\title{
NONNEGATIVE MATRIX FACTORIZATION WITH ENDMEMBER SPARSE GRAPH LEARNING FOR HYPERSPECTRAL UNMIXING
}

\author{
Bin Qian ${ }^{1}$, Jun Zhou ${ }^{2}$, Lei Tong ${ }^{3}$, Xiaobo Shen ${ }^{1}$, Fan Liu ${ }^{4}$ \\ ${ }^{1}$ School of Computer Science and Engineering, Nanjing University of Science and Technology, \\ Nanjing, 210094, Jiangsu, China \\ ${ }^{2}$ School of Information and Communication Technology, Griffith University, Nathan, QLD 4111, Australia \\ ${ }^{3}$ School of Engineering, Griffith University, Nathan, QLD 4111, Australia \\ ${ }^{4}$ College of Computer and Information, Hohai University, Nanjing, 210094, Jiangsu, China
}

\begin{abstract}
Nonnegative matrix factorization (NMF) based hyperspectral unmixing aims at estimating pure spectral signatures and their fractional abundances at each pixel. During the past several years, manifold structures have been introduced as regularization constraints into NMF. However, most methods only consider the constraints on abundance matrix while ignoring the geometric relationship of endmembers. Although such relationship can be described by traditional graph construction approaches based on k-nearest neighbors, its accuracy is questionable. In this paper, we propose a novel hyperspectral unmixing method, namely NMF with endmember sparse graph learning, to tackle the above drawbacks. This method first integrates endmember sparse graph structure into NMF, then simultaneously performs unmixing and graph learning. It is further extended by incorporating abundance smoothness constraint to improve the unmixing performance. Experimental results on both synthetic and real datasets have validated the effectiveness of the proposed method.
\end{abstract}

Index Terms - Hyperspectral unmixing, Nonnegative matrix factorization, Sparse Graph Learning, Manifold regularization, K-nearest neighbors.

\section{INTRODUCTION}

Hyperspectral remote sensing imagery contains both spatial and spectral information of land covers [1]. Most hyperspectral images consists of pixels with both pure and mixed reflectance from ground objects due to its limited spatial resolution. Hence, hyperspectral unmixing is often employed as a necessary preprocessing step to decompose each mixed pixel into a collection of constituent spectra, or endmembers, and their corresponding fractional abundances $[2,3]$.

Linear unmixing model, which is widely used for hyperspectral unmixing, assumes that distinct endmembers are not

\footnotetext{
${ }^{1}$ This work was supported by the Fundamental Research Funds for the Central Universities of China[Grant No.2015B03114]
}

interfered with each other. Nonnegative matrix factorization (NMF) [4] is one of the solutions based on linear mixing model. It simultaneously estimates both endmembers and their abundance without a predefined spectral library. NMF solution can easily fall into local minima, which is caused by the non-convexity of its objective function [5]. To alleviate this drawback, several constraints have been imposed on NMF, for example, piecewise smoothness over abundance data [6], endmembers dissimilarity [7], and abundance sparsity [8,9].

Geometric structure of the data is also considered. Lu et al. [10] proposed a graph regularized NMF (GLNMF) method by imposing both manifold regularization and sparseness constraints on the abundance matrix. Later on, based on this approach, Tong et al. [11] developed a multiple graph manifold NMF (MNMF) to model the multi-scale geometrical structure of abundance. However, although promising in performance, these NMF methods only make constraints on abundance matrix, and neglect the correlation of endmembers. Furthermore, traditional way of using k-nearst neighbor (KNN) for graph construction in these methods fails to precisely describe the relationship between endmembers. To provide a better way of graph construction, sparsity based graph learning has received more and more attention $[12,13]$.

In this paper, a novel method, namely NMF with endmember sparse graph learning (ESGNMF) is proposed for hyperspectral unmixing. ESGNMF integrates a sparse graph learning model with NMF to recursively learn the similarity between endmembers. It not only helps to discover the intrinsic data structure in the low dimensionality feature space, but also completes the matrix factorization task simultaneously. We further extend ESGNMF by incorporating abundance smoothness to improve the unmixing performance.

\section{RELATED WORK}

In this section, we briefly introduce the NMF based hyperspectral linear unmixing method. Given a hyperspectral image data $X \in \mathbb{R}_{+}^{L \times N}$, each column of $X$ is a pix- 
el which contains $L$ spectral bands. NMF aims to find two nonnegative matrices $A \in \mathbb{R}_{+}^{L \times K}$ (endmember) and $S \in \mathbb{R}_{+}^{K \times N}$ (abundance) by minimizing the following objective function:

$$
\begin{aligned}
& \min _{A, S} F(A, S)=\frac{1}{2}\|X-A S\|_{F}^{2} \\
& \text { s.t. } A \geq 0, S \geq 0
\end{aligned}
$$

where $\|.\|_{F}$ denotes the matrix Frobenius norm and $K$ is the number of endmembers.

The above problem can be solved by a multiplied iterative algorithm, which consists of two steps:

$$
\begin{gathered}
A \leftarrow A . * X S^{T} \cdot / A S S^{T} \\
S \leftarrow S . * A^{T} X . /\left(A^{T} A S\right)
\end{gathered}
$$

where $(.)^{T}$ is the transpose of a matrix, .* and ./ denote element-wise multiplication and division, respectively. It is proved that after a few iterations the objective function in equation (1) can converge to a local optimum [5]. However, the results from NMF are unstable and prone to noise, so extra constraints should be considered for hyperspectral unmixing.

\section{APPROACH}

In this section, we first briefly describe the endmember sparse graph learning model [13] and then show how it can be used for hyperspectral unmixing. This is the first time that this model is introduced for hyperspectral unmixing problem. Then we present the proposed method and its corresponding iterative solution.

\subsection{Endmember Sparse Graph Learning}

The traditional KNN-based graph construction can be directly applied to model the spatial relationship between pixels in abundance estimation. However, it is not suitable for modeling the relationship between endmembers due to their arbitrariness in spatial distribution. To tackle this problem, we introduce a nonnegative sparse graph learning model [13]. This model enables automatic characterization of the similarities among all endmembers, using the following objective function:

$$
\begin{aligned}
& \min _{W}\|A-A W\|_{F}^{2}+\lambda \operatorname{tr}(\Xi(A \odot M)) \\
& \text { s.t. } W \geq 0, W_{i i}=0 \forall i
\end{aligned}
$$

where $M_{i, j}=\left\|A_{* i}-A_{* j}\right\|_{F}^{2}$ and $A_{* i}$ denotes the $i$ th column of $A$. $\Xi \in \mathbb{R}^{n \times n}$ is a matrix with all elements as 1 and $\odot$ is a Hadamard product operator of matrices.

The second term in equation (4) ensures that each endmember be approximated by a linear combination of its nearby endmembers in the whole dataset and results in a locality

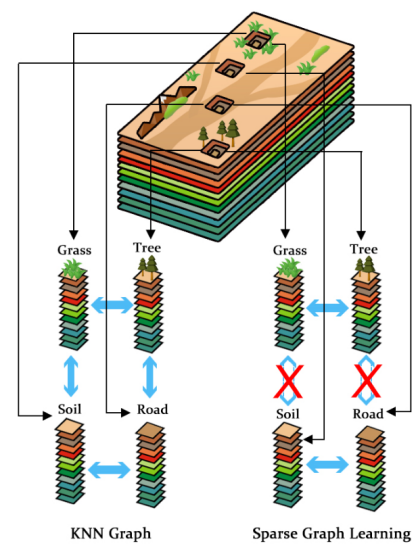

Fig. 1. Endmember graph construction based on KNN and sparse graph learning in hyperspectral scene.

driven solution. Unfortunately, this term leads to high computational cost and is difficult to be applied to NMF. Recently some researchers have shown that weight matrices in most locality-based dimensionality reduction methods, such as locality preserved projection (LPP) [14] and neighborhood preserving embedding (NPE) [15], are sparse in nature. Such sparsity is an important way to encode the domain knowledge, thus is helpful in improving the generalization capability of the model. Motivated by this characteristics, we replace the second term with a sparsity penalty term $\|W\|_{1 / 2}$, through which the sparsity can be naturally derived which makes the problem much easier to handle. In this way, the corresponding matrix factorization task becomes

$$
\begin{aligned}
& \min _{W}\|A-A W\|_{F}^{2}+\lambda\|W\|_{1 / 2} \\
& \text { s.t. } W \geq 0, W_{i i}=0 \forall i
\end{aligned}
$$

In order to integrate sparse graph learning and NMF into a unified iterative framework that helps to find the overall optimum, we further relax the constraints and propose the following objective function

$$
\begin{aligned}
& \min _{A, S, W} \frac{1}{2}\|X-A S\|_{F}^{2}+\lambda_{1}\|A-A W\|_{F}^{2}+\lambda_{2}\|W\|_{1 / 2} \\
& \text { s.t. } A \geq 0, S \geq 0, W \geq 0
\end{aligned}
$$

In this model, the endmember graph learning and NMF are simultaneously completed within one step for hyperspectral unmixing. Fig. 1 depicts the concept of the KNN graph construction method and the sparse graph learning method in a hyperspectral scene. There are four endmembers appearing in the simulation scene: grass, tree, soil and road. The response of grass is close to tree and the response of soil is close to road. As we can see that if we choose the wrong number of neighbors, e.g. 2 in the figure, the KNN graph cannot describe the relationship of endmembers accurately (this 
is not properly explained. Low weights will be given to the edge if two endmembers are not similar). Nevertheless, sparse graph learning method can model the correlation of endmembers in a global view and automatically decide the neighbors and weights. Therefore, it is beneficial to develop a novel graph construction method which can reflect the intrinsic geometric structure of endmembers in a robust and automatic way.

\subsection{ESGNMF}

Previous studies [10] have shown that abundance data are more likely to lie on a low-dimensional submanifold embedded in the high-dimensional ambient space. Thus, it is necessary to consider the intrinsic manifold structure of abundance for hyperspectral unmixing. We extend our method to further incorporate an abundance manifold regularization to exploit the latent manifold data structure during the decomposition.

Given an abundance graph $G=\{X, E\}$, where $E$ denotes a weighted matrix, the elements in $E$ can be defined as:

$$
E_{i j}= \begin{cases}1 & \text { if } x_{i} \in \mathrm{N}_{p}\left(x_{j}\right) \text { or } x_{j} \in \mathrm{N}_{p}\left(x_{i}\right) \\ 0 & \text { otherwise }\end{cases}
$$

where $\mathrm{N}_{p}\left(x_{j}\right)$ is the set of $p$ nearest neighbors of $x_{j}, L=$ $D-E$ is the Laplacian matrix, $D$ is a diagonal matrix and $D_{i i}=\sum_{j} E_{i j}$.

By incorporating an abundance manifold regularizer, we obtain the objective function of ESGNMF as follows:

$$
\begin{aligned}
& \min _{A, S, W} F=\frac{1}{2}\|X-A S\|_{F}^{2}+\frac{\lambda_{1}}{2}\|A-A W\|_{F}^{2}+\lambda_{2}\|W\|_{1 / 2} \\
& +\frac{\lambda_{3}}{2} \operatorname{Tr}\left(S L S^{T}\right) \quad \text { s.t. } A \geq 0, S \geq 0, W \geq 0
\end{aligned}
$$

Let $\psi_{i k}, \phi_{k j}$, and $\theta_{k m}$ be the Lagrange multiplers for constraints $A_{i k} \geq 0, S_{k j} \geq 0$ and $W_{k m} \geq 0$, respectively. We can update $A, S$ and $W$ iteratively by fixing the other parameters. Taking the partial derivatives of Lagrange $F$ over $A, S$ and $W$ of equation (8) as follows

$$
\begin{gathered}
\frac{\partial F}{\partial A}=-X S^{T}+A S S^{T}+\lambda_{1}\left(A-A W-A W^{T}+A W W^{T}\right)+\Psi \\
\frac{\partial F}{\partial S}=-A^{T} X+A^{T} A S+\lambda_{3} S(D-E)+\Phi \\
\frac{\partial F}{\partial W}=\lambda_{1}\left(-A^{T} A+A^{T} A W\right)+\frac{\lambda_{2}}{2} W^{-\frac{1}{2}}+\Theta
\end{gathered}
$$

then using KKT conditions $\psi_{i k} A_{i k}=0, \phi_{k j} S_{k j}=0$ and $\theta_{k m} W_{k m}=0$, we derive the following updata rules:

$$
\begin{gathered}
A \leftarrow A . * \frac{X S^{T}+\lambda_{1} A W+\lambda_{1} A W^{T}}{A S S^{T}+\lambda_{1} A+\lambda_{1} A W W^{T}} \\
S \leftarrow S . * \frac{A^{T} X+\lambda_{3} S E}{A^{T} A S+\lambda_{3} S D}
\end{gathered}
$$

$$
W \leftarrow W . * \frac{\lambda_{1} A^{T} A}{\lambda_{1} A^{T} A W+\frac{\lambda_{2}}{2} W^{-\frac{1}{2}}}
$$

\subsection{Implementation Issues}

During the unmixing process, the abundance at each pixel should sum to one. To this end, we adopt the solution in [16]. The data matrix $X$ and the signature matrix $A$ in equation (12) are replaced by $X_{f}$ and $A_{f}$ which are augmented matrices by a row of constants defined as follows

$$
X_{f}=\left(\begin{array}{c}
X \\
\delta 1_{N}^{T}
\end{array}\right) A_{f}=\left(\begin{array}{c}
A \\
\delta 1_{K}^{T}
\end{array}\right)
$$

where the parameter $\delta$ is used to balance the impact of the additivity constraint on the abundance matrix. A large $\delta$ forces the sum of abundances at each pixel closer to unity.

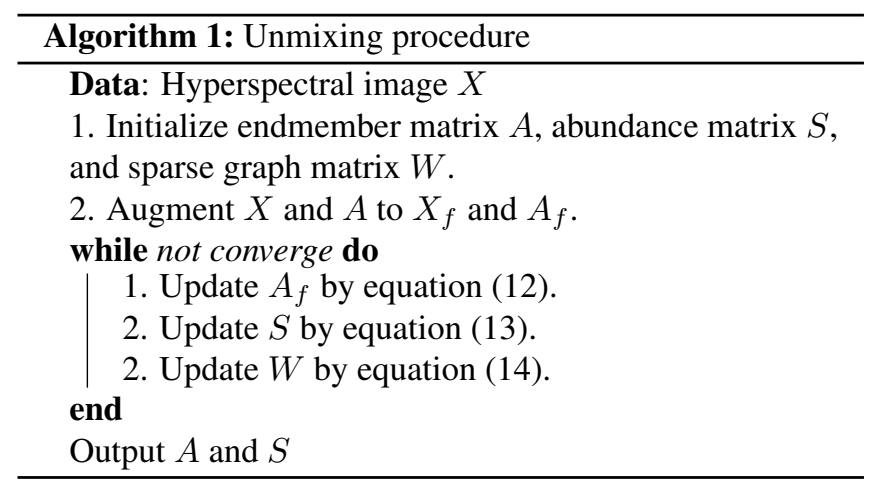

As shown in Algorithm 1, the computation mainly costs in the iterative procedure. if the iteration stops after $t$ steps, the total cost of ESGNMF is $O\left(t L K N+t K N^{2}\right)$.

\section{EXPERIMENTS}

We adopt both synthetic and real-world datasets to evaluate the proposed method. VCA [17], NMF [4], $L_{1 / 2}$-NMF [8], GLNMF [10] are chosen as the benchmark methods. We use the spectral angle distance (SAD) to compare the similarity of the end-member signature and its estimation, and the root mean squared error (RMSE) [10] to evaluate the abundance estimation.

\subsection{Experiments on Synthetic Data}

We first use spectral signatures chosen from the USGS digital spectral library [18] to build the synthetic dataset. The synthetic image is generated in a similar way as [10]. The image contains $64 \times 64$ pixels, which is divided into $8 \times 8$ regions. In each region the same type of ground cover is initialized with one of the endmembers chosen randomly. Then a low pass filter of size $9 \times 9$ is applied to generate mixed data and a threshold is used to increase the extent of mixing. Finally, 


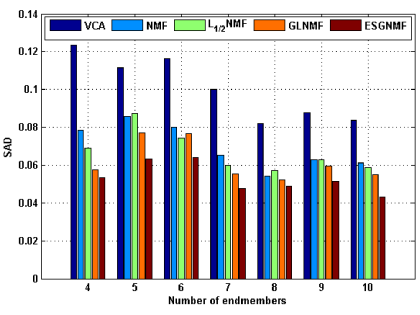

(a)

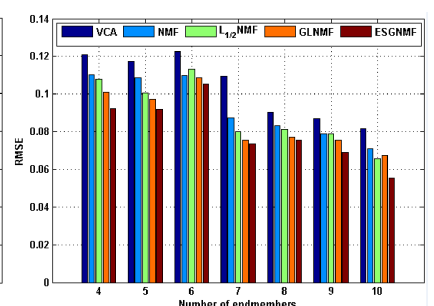

(b)
Fig. 2. Results of increasing the number of known endmembers. (a) Mean SADs; (b) Mean RMSEs.

Table 1. The result of different SNR

(a) SAD

\begin{tabular}{cccccc}
\hline SNR & VCA & NMF & $L_{1 / 2}$ NMF & GLNMF & ESGNMF \\
\hline 15 & 0.0974 & 0.1055 & 0.0921 & 0.0883 & $\mathbf{0 . 0 8 7 4}$ \\
20 & 0.1096 & 0.0935 & 0.0820 & 0.0877 & $\mathbf{0 . 0 8 1 3}$ \\
25 & 0.1165 & 0.0799 & 0.0745 & 0.0766 & $\mathbf{0 . 0 6 4 0}$ \\
30 & 0.1144 & 0.0546 & 0.0617 & 0.0573 & $\mathbf{0 . 0 5 4 0}$ \\
35 & 0.1188 & 0.0531 & 0.0498 & 0.0490 & $\mathbf{0 . 0 4 6 9}$ \\
40 & 0.1186 & 0.0457 & 0.0462 & 0.0443 & $\mathbf{0 . 0 4 2 2}$ \\
Mean & 0.1125 & 0.0720 & 0.0677 & 0.0672 & $\mathbf{0 . 0 6 2 6}$
\end{tabular}

(b) RMSE

\begin{tabular}{cccccc}
\hline SNR & VCA & NMF & $L_{1 / 2}$ NMF & GLNMF & ESGNMF \\
\hline 15 & $\mathbf{0 . 1 0 6 7}$ & 0.1288 & 0.1196 & 0.1134 & 0.1105 \\
20 & 0.1191 & 0.1148 & 0.1136 & 0.1140 & $\mathbf{0 . 1 0 9 7}$ \\
25 & 0.1228 & 0.1099 & 0.1132 & 0.1088 & $\mathbf{0 . 1 0 5 6}$ \\
30 & 0.0788 & 0.0671 & 0.0723 & 0.0644 & $\mathbf{0 . 0 6 4 2}$ \\
35 & 0.0849 & 0.0633 & 0.0621 & 0.0556 & $\mathbf{0 . 0 5 5 0}$ \\
40 & 0.0852 & 0.0457 & 0.0471 & 0.0452 & $\mathbf{0 . 0 4 5 5}$ \\
Mean & 0.0996 & 0.1029 & 0.0879 & 0.0835 & $\mathbf{0 . 0 8 1 8}$ \\
\hline
\end{tabular}

Gaussian noise is added to the synthetic data. The signal-tonoise ratio(SNR) can be defined as:

$$
S N R=10 \log _{10} \frac{E\left[x^{T} x\right]}{e^{T} e}
$$

where $x$ and $e$ represent the observation and noise at a pixel and $E[\cdot]$ denotes the expectation operator.

The first experiment evaluates the influence of different number of endmembers. We increase the endmembers from 4 to 10 and randomly generate the synthetic data. Here we set the parameters as follows: $\lambda_{1}=0.2, \lambda_{2}=0.1, \lambda_{3}=$ $0.05, S N R=25$. The random experiments are repeated 20 times and the average performance is then taken as the final result. Fig. 2 shows the unmixing results of different methods. From this figure, we can clearly see that the proposed method achieves the best performance among all methods.

In the second experiment, we evaluate the robustness of the proposed method under different noises. Table 1 shows the results of all methods under different SNR. It can been found that SADs and RMSEs of the proposed method are superior to other methods in most cases.

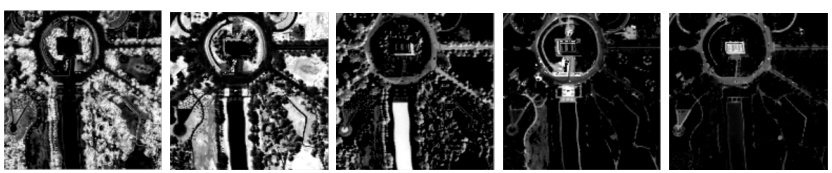

Fig. 3. Results of abundance estimation. From left to right: tree, grass, water, roof, and street.

\subsection{Experiments on Remote Sensing Data}

The last experiment is conducted on the Washington DC Mall dataset captured by the Urban Hyperspectral Digital Imagery Collection Experiment (HYDICE) sensor. Due to the large size, we crop the original image to a sub-image with the size of $150 \times 150$. Only 191 bands remain after removing lowSNR bands. Here five endmembers, i.e., tree, grass, water, roof, and street are chosen for this experiment.

The setting of the parameters in this experiment is the same as the previous experiments. Fig. 3 shows the results of abundance estimation on the Washington DC Mall data. From the $1^{\text {th }}$ to the $5^{\text {th }}$ column, they are the abundances of tree, grass, water, roof, and street, respectively. The images are in grayscale, in which a dark pixel indicates that the abundance of a particular endmember is low, and vice versa. Table 2 shows the SAD of the endmember estimation with all the unmixing methods. The results demonstrate that the performance of the proposed method is better than the alternative methods.

Table 2. Comparison between methods in terms of SAD

\begin{tabular}{cccccc}
\hline Endmember & VCA & NMF & $L_{1 / 2}$ NMF & GLNMF & ESGNMF \\
\hline Tree & 0.1946 & 0.2223 & 0.1874 & 0.1638 & $\mathbf{0 . 1 5 4 0}$ \\
Grass & 0.1845 & 0.1907 & 0.1565 & 0.1349 & $\mathbf{0 . 1 2 5 8}$ \\
Water & 0.1077 & 0.1138 & 0.0790 & $\mathbf{0 . 0 6 5 4}$ & 0.0665 \\
Roof & 0.1542 & 0.1499 & 0.1232 & 0.1179 & $\mathbf{0 . 1 0 0 3}$ \\
Street & 0.2146 & 0.1758 & 0.1663 & 0.1583 & $\mathbf{0 . 1 5 6 4}$ \\
Mean & 0.1711 & 0.1705 & 0.1425 & 0.1280 & $\mathbf{0 . 1 2 0 6}$ \\
\hline
\end{tabular}

\section{CONCLUSION}

In this paper, a novel method named nonnegative matrix factorization with endmember sparse graph learning (ESGNMF) is introduced for hyperspectral unmixing. The matrix factorization step in the unmixing process can be accurately guided by the endmember sparse graph structure which helps not only to discover the intrinsic data structure but also to fit the factorization mission well. Then ESGNMF is further extended by incorporating abundance smoothness constraint to take full advantage of the hyperspectral data and improve the unmixing result. Compared with the state-of-the-art methods, the superiority of the proposed method in hyperspectral unmixing has been validated on both synthetic and real datasets. 


\section{REFERENCES}

[1] Z. Zou, Z. Shi, J. Wu, and H. Wang, "Quadratic constrained energy minimization for hyperspectral target detection," in Proceedings of IEEE International Geoscience and Remote Sensing Symposium, 2015, pp. 4979-4982.

[2] J.M. Bioucas-Dias, A. Plaza, N. Dobigeon, M. Parente, Q. Du, P. Gader, and J. Chanussot, "Hyperspectral unmixing overview: Geometrical, statistical, and sparse regression-based approaches," IEEE Journal of Selected Topics in Applied Earth Observations and Remote Sensing, vol. 5, no. 2, pp. 354-379, 2012.

[3] W. Tang, Z. Shi, Y. Wu, and C. Zhang, "Sparse unmixing of hyperspectral data using spectral a priori information," IEEE Transactions on Geoscience and Remote Sensing, vol. 53, no. 2, pp. 770-783, 2015.

[4] V. Pauca, J. Piper, and R. Plemmons, "Nonnegative matrix factorization for spectral data analysis," Linear algebra and its applications, vol. 416, no. 1, pp. 29-47, 2006.

[5] D. Cai, X. He, J. Han, and T.S. Huang, "Graph regularized nonnegative matrix factorization for data representation," IEEE Transactions on Pattern Analysis and Machine Intelligence, vol. 33, no. 8, pp. 1548-1560, 2011.

[6] S. Jia and Y. Qian, "Constrained nonnegative matrix factorization for hyperspectral unmixing," IEEE Transactions on Geoscience and Remote Sensing, vol. 47, no. 1, pp. 161-173, 2009.

[7] N. Wang, B. Du, and L. Zhang, "An endmember dissimilarity constrained non-negative matrix factorization method for hyperspectral unmixing," IEEE Journal of Selected Topics in Applied Earth Observation and Remote Sensing, vol. 6, no. 2, pp. 554-569, 2013.

[8] Y. Qian, S. Jia, J. Zhou, and A. Robles-Kelly, "Hyperspectral unmixing via $L_{1 / 2}$ sparsity-constrained nonnegative matrix factorization," IEEE Transactions on Geoscience and Remote Sensing, vol. 49, no. 11, pp. 4282-4297, 2011.

[9] W. Wang and Y. Qian, "Adaptive 11/2 sparsityconstrained nmf with half-thresholding algorithm for hyperspectral unmixing," IEEE Journal of Selected Topics In Applied Earth Observations And Remote Sensing, vol. 8, no. 6, pp. 2618-2631, 2015.

[10] X. Lu, H. Wu, Y. Yuan, P. Yan, and X. Li, "Manifold regularized sparse nmf for hyperspectral unmixing," IEEE Transactions on Geoscience and Remote Sensing, vol. 51, no. 5, pp. 2815-2126, 2013.
[11] L. Tong, J. Zhou, Y. Qian, and Y. Gao, "Multiple graph regularized nmf for hyperspectral unmixing," in Proceedings of the 7th Workshop on Hyperspectral Image and Signal Processing: Evolution in Remote Sensing, 2015, pp. 1-4.

[12] J. Huang, F. Nie, and H. Huang, "A new simplex sparse learning model to measure data similarity for clustering," in Proceedings of the 24th International Conference on Artificial Intelligence, 2015, pp. 3569-3575.

[13] X. Fang, Y. Xu, X. Li, Z. Lai, and W. Wong, "Learning a non-negative sparse graph for linear regression," IEEE Transactions on Image Processing, vol. 24, no. 9, pp. 2760-2771, 2015.

[14] X. Niyogi, "Locality preserving projections," in Neural information processing systems, 2004, vol. 16, p. 153.

[15] X. He, D. Cai, S. Yan, and H. Zhang, "Neighborhood preserving embedding," in IEEE International Conference on Computer Vision, 2005, vol. 2, pp. 1208-1213.

[16] D. Heinz and C. Chang, "Fully constrained least squares linear spectral mixture analysis method for material quantification in hyperspectral imagery," IEEE Transactions on Geoscience and Remote Sensing, vol. 39, no. 3, pp. 529-545, 2001.

[17] J. Nascimento and J. Dias, "Vertex component analysis: A fast algorithm to unmix hyperspectral data," IEEE Transactions on Geoscience and Remote Sensing, vol. 43, no. 4, pp. 898-910, 2005.

[18] R. Clark, G. Swayze, R. Wise, K.E Livo, T.M Hoefen, R.F Kokaly, and S.J. Sutley, "USGS digital spectral library splib06a: U.S. Geological Survey, Digital Data Series 231," USGS, 2007. 